Abstract

\title{
Application of SORTS, a Novel Gene-Edited Cell Selection Method for HIV Study and Therapy ${ }^{\dagger}$
}

\author{
Dmitriy Mazurov ${ }^{1,2, *}$, Alexandra Maslennikova ${ }^{1}$, Dmitriy Komkov ${ }^{1}$ and Anastasia Zotova ${ }^{1}$ \\ 1 Cell and Gene Technology Group, Institute of Gene Biology RAS, Moscow 119334, Russia; \\ a.k.dorogush@gmail.com (A.M.); dmitkomserg@gmail.com (D.K.); ashunaeva@gmail.com (A.Z.) \\ 2 NRC Institute of Immunology FMBA of Russia, Moscow 115478, Russia \\ * Correspondence: dvmazurov@yandex.ru \\ † Presented at Viruses 2020-Novel Concepts in Virology, Barcelona, Spain, 5-7 February 2020.
}

Published: 4 June 2020

\begin{abstract}
We have recently developed surface oligopeptide knock-in for rapid target selection (SORTS), a novel method to isolate mammalian cells with gene modifications using FACS-sorting. It relies on CRISPR/Cas9-mediated targeted knock-in of a very short promoterless expression construct (250 bp) comprising a Flag or HA epitope embedded into the smallest GPI-protein CD52, and a polyA signal from the beta-globin. CD52 efficiently delivers the epitope to the cell surface, where it serves as a marker for selection, while polyA terminates transcription and silences target gene expression. Primarily, SORTS was developed to knock out genes encoding intracellular or secreted proteins, which cannot be used as markers for selection of live cells. Using in-frame modification of SORTS, we demonstrated the possibility of HIV-1 provirus inactivation via sorting of GPI-tag positive cells. In order to make the "cured" cells resistant to a subsequent HIV-1 infection, the epitope tag in the CD52 molecule was substituted by one of the fusion inhibitor peptides from the CHR-domain of gp41. We selected a series of cell-surface-expressed, GPI-anchored, C34-based peptides that confer a strong cellular resistance to HIV-1 infection mediated by NL4-3, JRFL, or ZM153 Env. These findings together with a monoclonal antibody raised against the $\mathrm{C} 34$ peptide provide an opportunity to generate and select HIV-resistant lymphocytes for a therapeutic goal. SORTS was also adapted to engineer transgenic HIV-1 effector $\mathrm{T}$ cells and to study cell-to-cell transmission. To facilitate transgenesis, we developed a knock-in strategy to express GPI-tag from the intronic region of the human PPP1R12C gene (AAVS1 locus) and delivered FRT sites of recombination into both alleles. In summary, SORTS is a novel instrument to isolate rare cells with precise genomic modifications with broad applications, including HIV biology. This work was supported by the Russian Science Foundation (grant 18-14-00333) and the Russian Foundation for Basic Research (grants 18-29-07052, 18-04-01016).
\end{abstract}

Keywords: SORTS; CD52; HIV-1; gene editing; CRISPR-Cas9; fusion inhibitor peptides

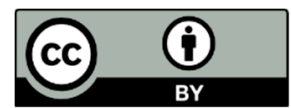

(C) 2020 by the authors. Licensee MDPI, Basel, Switzerland. This article is an open access article distributed under the terms and conditions of the Creative Commons Attribution (CC BY) license (http://creativecommons.org/licenses/by/4.0/). 